\title{
Relationship between STarT Back Screening Tool and prognosis for low back pain patients receiving spinal manipulative therapy
}

\author{
Jonathan Field ${ }^{1 *}$ and Dave Newell ${ }^{2}$
}

\begin{abstract}
Background: Low back pain (LBP) is common and costly and few treatments have been shown to be markedly superior to any other. Effort has been focused on stratifying patients to better target treatment. Recently the STarT Back Screening Tool (SBT) has been developed for use in primary care to enable sub grouping of patients based on modifiable baseline characteristics and has been shown to be associated with differential outcomes. In the UK the SBT is being recommended to assist in care decisions for those presenting to general practitioners with LBP. In the light of growing recommendation for widespread use of this tool, generalisability to other LBP populations is important. However, studies to date have focused only on patients attending physiotherapy whereas LBP patients seeking other treatment have not been investigated.
\end{abstract}

Aims: This study aims to investigate the utility of the SBT to predict outcomes in LBP patients presenting for chiropractic management.

Methods: A total of 404 patients undergoing chiropractic care were asked to complete the SBT before initial treatment. Clinical outcomes were collected at 14, 30 and 90 days following this initial consultation. The clinical course was described comparing SBT categories and logistic regression analysis performed to examine the tool's prognostic utility.

Results: Although the high-risk categories had greater pain at baseline this difference rapidly faded, with both change in composite outcome scores and pain scores being statistically insignificant between the risk groups at 30 and 90 days follow up. In addition, both univariate and adjusted analysis showed no prognostic utility of the SBT categorisations to differentiate clinical outcomes between risk groups.

Conclusion: Whilst the SBT appears useful in some back pain populations it does not appear to differentiate outcomes in LBP patients seeking chiropractic care.

Keywords: Start back tool, Low back pain, Prognosis, Spinal manipulative therapy

\section{Introduction}

Low back pain (LBP) is a common symptom causing health-seeking behavior in up to half of those who experience it [1,2]. Between six and nine percent of the UK population consult their general practitioner (GP) for LBP each year, accounting for 5 million GP consultations annually $[3,4]$. For most of these patients a low back pain episode will most likely be a temporary inconvenience, yet

\footnotetext{
* Correspondence: jonathanfield@me.com

'Private practice Back2Health, 2 Charles Street, Petersfield, Hants, UK

Full list of author information is available at the end of the article
}

for a minority who have sought care (approximately 28\%) it becomes an enduring and disabling problem $[5,6]$.

The extent of the costs to society of this syndrome have led to the call for identification of potential subgroups of non specific low back pain (nsLBP) in the belief that this group consists of a heterogeneous mix of presentations and etiologies. Identification of groups of back pain that respond better with specific interventions would facilitate targeted treatment [7]. In addition, evidence-based guidelines highlight the need to consider prognostic factors when deciding the management of nsLBP [8-10], where early identification of potential

\section{Biomed Central}


barriers to recovery may help guide treatment aimed at secondary prevention of persistent back pain $[11,12]$.

In the absence of serious pathology, recovery from back pain in individuals from the general back pain population as well as those seeking help from chiropractors is only weakly related to physical findings [13-17], with only a small number of condition specific factors associated with poor prognosis $[13,18,19]$. However, psychological factors are found to influence future disability, pain and self reported improvement in LBP patients presenting to GPs, secondary care services and surgery [20-23]. This has led to guidelines recommending that non-physical factors be considered when setting the treatment for LBP patients [8-10]. In chiropractic LBP populations the significance of psychological factors is less certain as exploratory studies have found little or no correlation with outcomes [14,24-26].

The STarT Back Tool (SBT) has recently been developed to help primary care practitioners make care decisions about the likely need LBP patients have for secondary prevention based on modifiable risk factors for poor outcome [20]. The SBT places patients into one of three categories (Low, Medium and High) of risk for having persisting LBP with disability. In a recent trial, patients whose care had been stratified using the SBT to receive either advice alone, 'standardised' physiotherapy or psychologically based care with physiotherapy had lower disability at 4 and 12 months than those patients undergoing usual care as directed by the clinical judgment of a physiotherapist [27]. As a consequence of those studies the SBT is being recommended by commissioning services in the UK NHS to guide care pathways for those presenting to GP with LBP. Whilst the feasibility of using the SBT in a chiropractic patient population has been demonstrated [28], as yet, no appraisal of the prognostic utility of grouping individuals seeking chiropractic care has been published.

This paper investigated whether nsLBP patients classified in the high-risk (complex psychosocial) group by the SBT do less well with chiropractic care than those either at low risk or medium risk groups.

\section{Methods}

\section{Subjects and procedure}

Consecutive patients aged over 16 presenting with nsLBP to one of six chiropractic clinics in the south of England were asked, as part of normal practice to complete the Bournemouth Questionnaire (BQ) [29] either at the clinic or on-line before their first visit. Those doing so on line were additionally asked to complete the SBT. Patients were presented with a consent form when they completed pre-examination forms online, via a web page. In these practices patients who start treatment are emailed outcome assessment questionnaires consisting of the BQ and a Patients Global Impression of Change (PGIC), at 14, 30 and 90 days following their initial visit.

\section{Outcomes}

For this study, the primary outcome was the PGIC. In addition we also measured pain as derived from the pain sub-scale of the BQ and total BQ scores. Patients were categorised into the three SBT risk groups using the method as described by Hill et al. [20,30]. For each of the follow up points, all outcomes were dichotomized. Thus poor outcome was defined by a PGIC response of better or much better (score of $<6$ ) [31], a change in pain of less than or equal to $(\leq) 2$ points [32] and a change in total BQ of $\leq 46 \%$ [33]. Recently, both the PGIC and BQ have been recommended as preferred measurements by the Any Qualified Provider Resource Centre' (UK, NHS) for monitoring outcomes in low back pain patients [34].

\section{Analysis}

General characteristics of the patient sample were calculated as means and proportions with appropriate measures of variance. Differences between categorical baseline characteristics were determined using $\chi^{2}$, or Pearson $\chi^{2}$ test for trend. Further, a Kolmogorov-Smirnov test revealed significant deviation from Gaussian distributions for pain and total BQ data, despite using logarithmic transformation. Consequently differences within these variables across SBT categories were analysed using Kruskal Wallis tests, whereas change scores revealed a Gaussian distribution and were analysed using a one-way ANCOVA adjusted for baseline scores.

To determine any associations between baseline SBT categorisation and the outcomes univariate logistic regression analysis was carried out using the SBT categorisation as the independent variable and the dichotomised outcomes (PGIC, change in pain and change in total $\mathrm{BQ}$ ) as dependent variables at each of the follow up time points. This analysis was repeated after sub grouping by gender, but only for the PGIC as a dependent variable. Finally, adjusted models for predicting poor outcome as defined by the PGIC were constructed with an entry criterion for significant variables of $\mathrm{p}<0.15$ and retention at $\mathrm{p}<0.05$ using a binary logistic analysis forward LR procedure. All statistical analyses were performed using statistical software SPSS (v17.0, SPSS Inc., Chicago IL).

\section{Ethics}

Ethics for this study were sought and approved by the Research and Ethics subcommittee of the Anglo-European College of Chiropractic Research Committee.

\section{Results}

A total of 819 patients presented with nsLBP between March and November 2011. Four hundred and five were ineligible to participate in the study, as they did not complete 
the initial forms online. There was no difference $(p>0.05)$ in gender, duration, or $\mathrm{BQ}$ scores at presentation or in pain and BQ scores at 90 days follow up between those submitting the forms online and those doing so at the clinic. However at presentation those completing the forms online and so being included in this study had slightly less pain (mean (SD) 6.2(2.0) versus 6.6(2.2), p0.04) and were younger (44.7 (15) versus 48.9(15.4) years, $\mathrm{p}<0.01)$. Four hundred and four individuals completing the forms online were categorized by the SBT at the study inception. Of these 168 (41.6\%), 129 (31.9\%) and 107 (26.5\%) were at low, medium or high risk of poor outcome at baseline respectively. Numbers of participants at follow up within each SBT group and percentage response rates are shown in Figure 1. The number of treatments received across the three SBT categories were; low (4.1(2.4)); medium (4.3 (2.4)) and high (4.4 (2.6)). There were no significant differences in treatment visits between SBT categories $(\mathrm{p}=0.54)$.

Table 1 shows the demographic and condition specific characteristics within each SBT risk group. Only the proportions of those patients with pain above the knee, higher baseline pain and greater total $\mathrm{BQ}$ scores differed between the SBT categories. The distribution of duration of back pain in the complete sample was $56.2 \%, 12.4 \%$ and $31.4 \%$ for less than 1 month, 1-3 months and greater than 3 months respectively. These proportions were similar across the SBT risk groups.
Table 1 Descriptive analysis of baseline variables across Start Back Tool (SBT) categories

\begin{tabular}{|c|c|c|c|}
\hline \multirow[t]{2}{*}{ Variables } & \multicolumn{3}{|c|}{ SBT Category } \\
\hline & $\begin{array}{l}\text { Low } \\
(n=168)\end{array}$ & $\begin{array}{l}\text { Medium } \\
(\mathrm{n}=129)\end{array}$ & $\begin{array}{l}\text { High } \\
(n=107)\end{array}$ \\
\hline Mean age (SD) & $45.4(15.1)$ & $45.9(15.0)$ & $45.8(14.1)$ \\
\hline Female & $54.8 \%$ & $52.7 \%$ & $50.5 \%$ \\
\hline Seen practitioner before & $47.0 \%$ & $59.7 \%$ & $33.6 \%$ \\
\hline \multicolumn{4}{|l|}{ Leg Pain } \\
\hline Above the knee ${ }^{*}$ & $25.5 \%$ & $23.3 \%$ & $35.5 \%$ \\
\hline Below the knee & $6.0 \%$ & $10.9 \%$ & $12.1 \%$ \\
\hline$>30$ days pain in year & $41.1 \%$ & $41.1 \%$ & $38.3 \%$ \\
\hline Recurring & $69.6 \%$ & $65.9 \%$ & $59.8 \%$ \\
\hline \multicolumn{4}{|l|}{ Duration } \\
\hline$<1$ month & $54.8 \%$ & $53.5 \%$ & $61.7 \%$ \\
\hline 1-3 months & $14.3 \%$ & $14.0 \%$ & $7.5 \%$ \\
\hline$>3$ months & $31.0 \%$ & $32.6 \%$ & $30.8 \%$ \\
\hline Median Pain $(25,75)^{* *}$ & $5(4-7)$ & $7(6-8)$ & $7(6-9)$ \\
\hline Median BQ $(25,75){ }^{* *}$ & $24(14-33)$ & $36(30-44)$ & $45(34-54)$ \\
\hline
\end{tabular}

${ }^{*} \mathrm{P}<0.05$ (Chi ${ }^{2}$ test for trend). ${ }^{* *} \mathrm{p}<0.001$, (Kruskal-Wallis).

Table 2 shows the total $\mathrm{BQ}$ and pain scores over time for each of the SBT categories. Pain and total BQ scores differed significantly between the SBT risks groups at

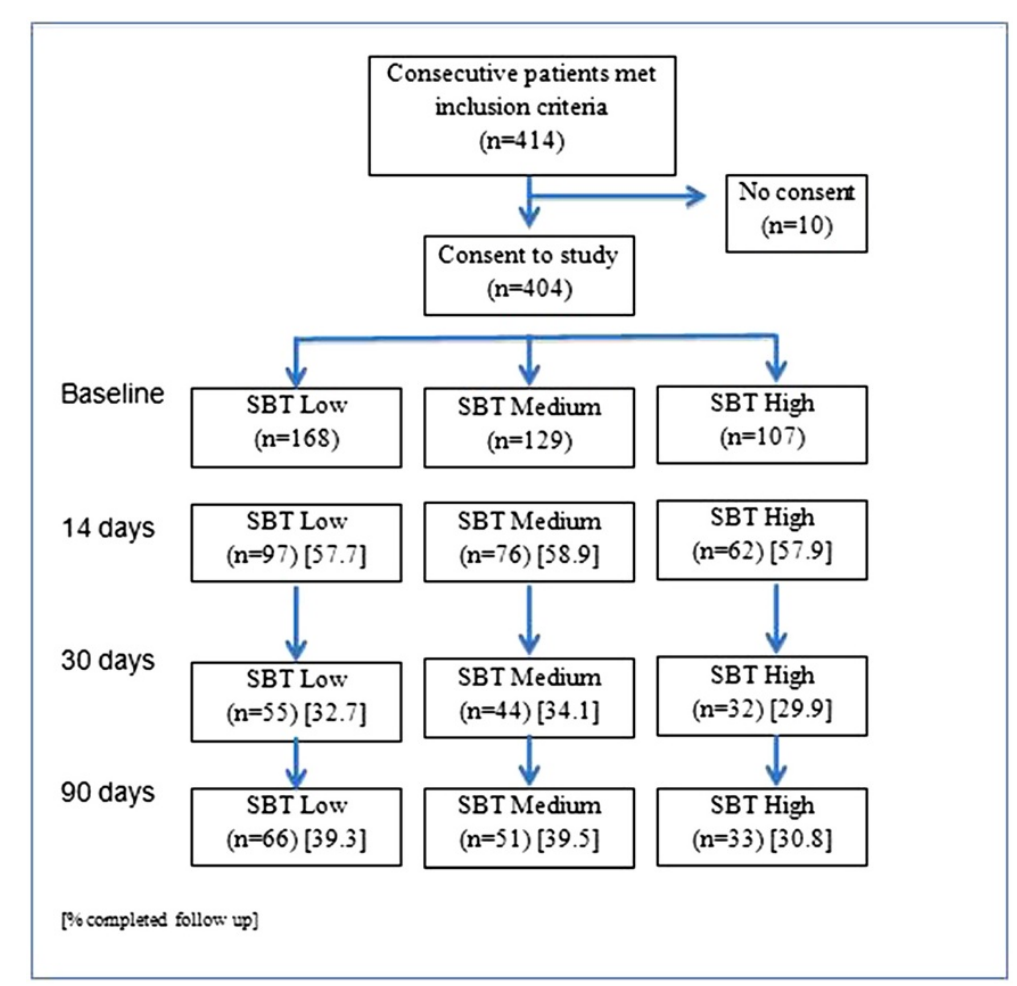

Figure 1 Numbers in study at each follow up point in each Start Back Tool category. 
Table 2 Pain, total BQ scores and proportion of subjects with a poor outcome across Start Back Tool (SBT) categories at 14,30 and 90 days follow up

\begin{tabular}{|c|c|c|c|c|c|c|}
\hline \multirow[t]{2}{*}{ SBT Category } & \multicolumn{2}{|l|}{14 days $(n=235)$} & \multicolumn{2}{|l|}{30 days $(n=131)$} & \multicolumn{2}{|l|}{90 days $(n=150)$} \\
\hline & Median Pain $(25,75)$ & Median BQ $(25,75)^{* *}$ & Median Pain $(25,75)$ & Median BQ $(25,75)$ & Median Pain $(25,75)$ & Median BQ $(25,75)$ \\
\hline Low & $2(1-3)$ & $10(3-18)$ & $1(1-3)$ & $6(2-14)$ & $1(1-2)$ & $5(0-15)$ \\
\hline Medium & $2(1-5)$ & $12(3-26)$ & $2(1-4)$ & $10(5-21)$ & $1(1-4)$ & $8(0-18)$ \\
\hline High & $3(1-5)$ & $20(8-33)$ & $2(0-3)$ & $6(2-20)$ & $2(0-3)$ & $10(3-22)$ \\
\hline
\end{tabular}

${ }^{* *} \mathrm{p}<0.001$, (Kruskal-Wallis), ${ }^{*}$ Patients Global Impression of Change $<6$.

baseline, where high-risk groups started with higher scores $(\mathrm{p}<0.001)$. The total BQ scores also differed at 14 days follow up $(\mathrm{p}<0.001)$. However, any differences between the risk groups were absent by 30 days with all groups achieving very similar scores by 90 days. Mean residual change scores for total $\mathrm{BQ}$ and pain are illustrated in Figure 2. These changes are not significantly different between risk categories at any of the follow up time points (Change in Pain: 14 days (ANCOVA. $\mathrm{F}=1.1, \mathrm{p}=0.32$ ); 30 days (ANCOVA. $\mathrm{F}=1.4, \mathrm{p}=0.25$ ); 90 days (One way ANCOVA. $\mathrm{F}=0.5, \mathrm{p}=0.62$ ) Change in total $\mathrm{BQ}$ : 14 days (ANCOVA. $\mathrm{F}=0.8, \mathrm{p}=0.46$ ); 30 days (ANCOVA. $\mathrm{F}=0.2$, $\mathrm{p}=0.83$ ); 90 days (ANCOVA. $\mathrm{F}=0.2, \mathrm{p}=0.83$ )).

Table 3 shows the proportion of patients with poor outcome in each SBT group at each follow up point as defined by three dichotomized outcomes. A univariate

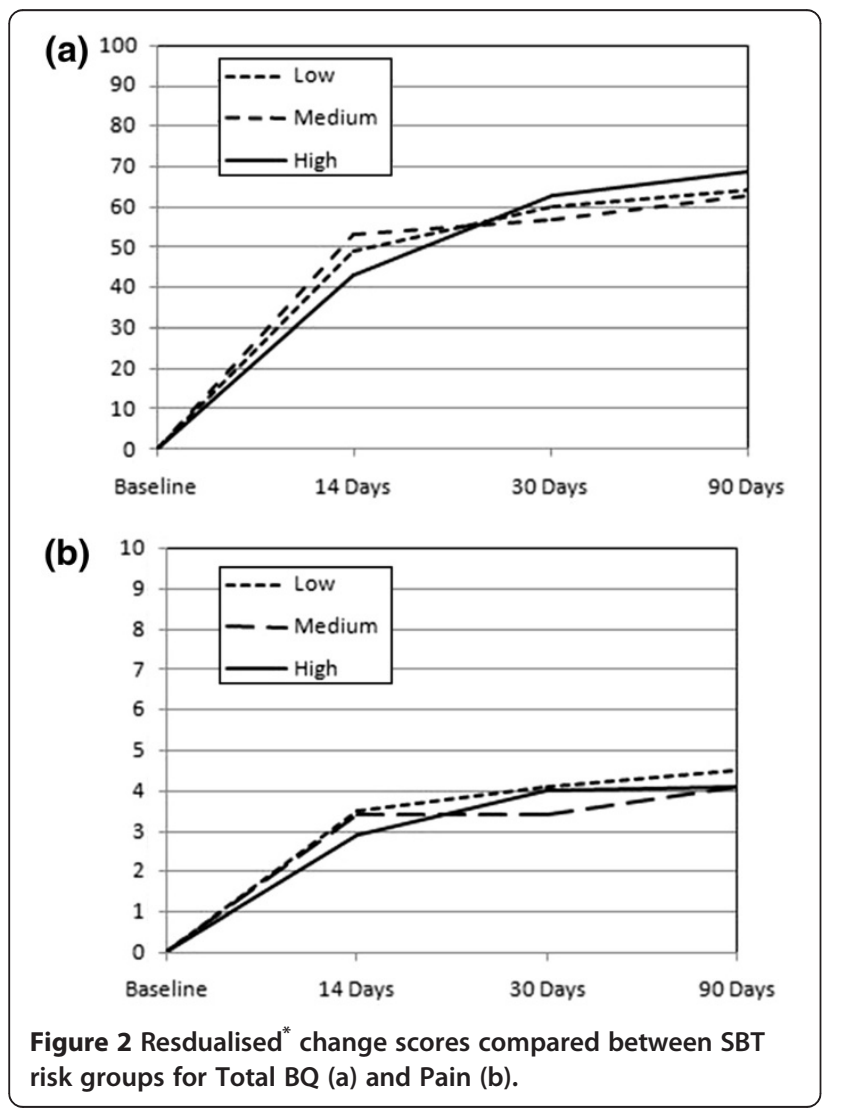

logistic regression analysis revealed that SBT categorisations were not statistically associated with the primary outcome (PGIC) at any of the follow up points (Table 4) However, the utility of the SBT to discriminate poor outcome using the PGIC did increase over time, with both medium and high risk groups being around 1.7 times the odds of poor outcome by 90 days compared to low risk groups, albeit not statistically significant. A further analysis using this outcome but sub grouping by gender revealed a similar, but again statistically insignificant association. In this analysis only male patients showed any marked association between baseline categorisation by SBT and poor outcome, where those in the medium and high risk groups had 3 times the odds of experiencing poor outcome compared to low risk groups at 90 days. In females however, the SBT had little predictive utility at any follow up point. (Table 5).

Categorising poor outcome as $\leq 2$ points drop in pain, a further analysis revealed no significant association between the SBT and an absence of meaningful change in pain at any follow up point (Table 6). This result was also apparent when using dichotomized total BQ change scores as the dependent variable (Table 7).

Table 3 Proportions (\%) of patients with a poor outcome across Start Back Tool categories defined by cut-off points for each outcome

\begin{tabular}{llll}
\hline Outcomes & $\mathbf{1 4}$ days $(\mathbf{n})$ & $\mathbf{3 0}$ days $(\mathbf{n})$ & $\mathbf{9 0}$ days $\mathbf{( n )}$ \\
\hline PGIC & & & \\
Low & $36.1(35)$ & $29.1(16)$ & $18.2(12)$ \\
Medium & $30.3(23)$ & $29.5(13)$ & $27.5(14)$ \\
High & $33.9(21)$ & $28.1(9)$ & $27.3(9)$ \\
$\leq \mathbf{2}$ on NRS & & & \\
Low & $37.9(33)$ & $24.0(12)$ & $23.0(14)$ \\
Medium & $24.7(18)$ & $26.2(11)$ & $24.5(12)$ \\
High & $32.8(19)$ & $32.3(10)$ & $18.8(6)$ \\
$\leq \mathbf{4 7 \%}$ on BQ & & & \\
Low & $28.9(24)$ & $20.4(10)$ & $14.0(8)$ \\
Medium & $29.4(20)$ & $25.6(10)$ & $19.6(9)$ \\
High & $41.5(22)$ & $29.0(9)$ & $18.2(6)$ \\
\hline
\end{tabular}

$P G / C=$ Patient Global Impression of Change; NRS = Numerical Rating Scale; $B Q=$ Bournemouth Questionnaire. 
Table 4 Predicting poor outcome (PGIC) at 14, 30 and 90 days for the whole group $(n=404)$

\begin{tabular}{|c|c|c|c|}
\hline \multirow{2}{*}{$\begin{array}{l}\text { SBT } \\
\text { Category }\end{array}$} & 14 days $(n=235)$ & 30 days $(n=131)$ & 90 days $(n=150)$ \\
\hline & OR $(95 \% \mathrm{Cl})$ & OR $(95 \% \mathrm{Cl})$ & OR $(95 \% \mathrm{Cl})$ \\
\hline Low & 1.0 & 1.0 & 1.0 \\
\hline Medium & 0.8 (0.4 to 1.5$)$ & $1.0(0.4$ to 2.4$)$ & 1.7 (0.7 to 4.1$)$ \\
\hline High & 0.9 (0.5 to 1.8$)$ & 0.9 (0.4 to 2.5$)$ & 1.7 (0.6 to 4.5$)$ \\
\hline
\end{tabular}

PGIC = Patient Global Impression of Change; $S B T=$ Start Back Tool.

A forward LR logistic analysis adjusting for all potential baseline predictors (Enter: $\mathrm{p} \leq 0.15$, Retain $\mathrm{p} \leq 0.05$ ) resulted in few variables remaining as independent predictors of outcome at the follow up points, with baseline SBT categorisations failing to be retained in any of the models (Table 8). Only duration, reoccurrence of the problem and pain for greater than 30 days in the last year provided any prognostic utility and even here the degree of variance explained remained relatively low as did the predictive accuracy.

\section{Discussion}

In this study those categorised by the SBT as being at low risk of having a poor prognosis were somewhat more likely to do well compared to those placed in the medium or high risk groups. However this difference was small and failed to reach any statistical significance in a univariate analysis. In addition the adjusted models at each follow up did not include the SBT tool as a significant predictor of outcome. This result suggests that, in this population at least, the proportion of high-risk patients that improve is not significantly different from those at medium or low risk.

Patients placed in the SBT high-risk group had more adverse $\mathrm{BQ}$ and pain scores at presentation. However, there was a greater improvement in these scores in the high-risk group with the result that by the 30 -day assessment this difference was no longer evident. These results are partially corroborated by Fritz et al. [35] who showed that despite high risk patients starting with more pain and disability at baseline they experienced greater improvement over a course of care.

The SBT has been developed to better stratify LBP patients for targeted treatment. Hill et al. (2008) [20]
Table 6 Predicting poor outcome (Change in pain $\leq 2$ points) at 14, 30 and 90 days

\begin{tabular}{|c|c|c|c|}
\hline \multirow{2}{*}{$\begin{array}{l}\text { SBT } \\
\text { Category }\end{array}$} & 14 days $(n=218)$ & 30 days $(n=123)$ & 90 days $(n=142)$ \\
\hline & OR $(95 \% \mathrm{Cl})$ & OR $(95 \% \mathrm{Cl})$ & OR $(95 \% \mathrm{Cl})$ \\
\hline Low & 1.0 & 1.0 & 1.0 \\
\hline Medium & 0.5 (0.3 to 1.1$)$ & $1.1(0.4$ to 2.9$)$ & 1.1 (0.4 to 2.6$)$ \\
\hline High & $0.8(0.4$ to 1.6$)$ & $1.5(0.5$ to 4.1$)$ & 0.8 (0.3 to 2.2) \\
\hline
\end{tabular}

SBT = Start Back Tool.

showed the discriminative ability of this tool to differentiate those who clinically changed on the RMDQ. This was largely based on differences in barriers to recovery, with the high-risk group being defined as having psychological barriers and the medium as having physical barriers. These authors went on to show that the SBT was able to support treatment choice more efficiently than physiotherapists' clinical experience alone. Although this approach has potential to improve outcomes and cost in LBP the generalisability of their results may not be supported by this study.

There may be a number of reasons why this is so. Firstly, this LBP population differs from previous studies in that they were largely self-selecting and sought chiropractic care privately. In addition to this, around $41 \%$ of this sample had experienced chiropractic care from the same practitioner previously. This may have led to higher expectations of success which may have impacted on the psychological response of people in this sample. Another possibility is that patients in the various SBT categories within our cohort study received different types of care and that this influenced their recovery. However, communicating with the participating clinicians indicated that they had not accessed the SBT category data, and there were no differences in the number of treatments provided between SBT categories. Despite this, differences in treatment approach cannot be ruled out. It is possible that those placed in the high risk SBT category received care that was consciously or unconsciously delivered by the chiropractor in a way so as to address the patient's psychological requirements. As a development of this idea, the treatment interventions in this study may inherently be similar to the treatment intervention given in Hill et al. (20) for their SBT high-

Table 5 Predicting poor outcome (PGIC) at 14, 30 and 90 days follow up split by gender

\begin{tabular}{|c|c|c|c|c|c|c|}
\hline \multirow{3}{*}{$\begin{array}{l}\text { SBT } \\
\text { Category }\end{array}$} & \multicolumn{2}{|l|}{14 days } & \multicolumn{2}{|l|}{30 days } & \multicolumn{2}{|l|}{90 days } \\
\hline & \multicolumn{2}{|l|}{ OR (95\% Cl) } & \multicolumn{2}{|l|}{ OR (95\% Cl) } & \multicolumn{2}{|l|}{ OR (95\% Cl) } \\
\hline & Male $(n=117)$ & Female $(n=118)$ & Male $(n=66)$ & Female $(n=65)$ & Male $(n=71)$ & Female $(n=79)$ \\
\hline Low & 1.0 & 1.0 & 1.0 & 1.0 & 1.0 & 1.0 \\
\hline Medium & 1.2 (0.5 to 3.0 ) & 0.5 (0.2 to 1.3$)$ & 0.7 (0.2 to 2.7$)$ & 1.2 (0.3 to 4.0$)$ & $3.0(0.9$ to 10.0$)$ & 0.9 (0.2 to 3.3$)$ \\
\hline High & 1.8 (0.7 to 5.0$)$ & 0.5 (0.2 to 1.2$)$ & 0.7 (0.2 to 3.2 ) & 1.0 (0.3 to 4.1$)$ & $3.0(0.6$ to 16.0$)$ & 1.1 (0.3 to 4.0$)$ \\
\hline
\end{tabular}

PGIC = Patient Global Impression of Change; SBT = Start Back Tool. 
Table 7 Predicting poor outcome (Change in total BQ $\leq 47 \%)$ at 14,30 and 90

\begin{tabular}{|c|c|c|c|}
\hline \multirow{2}{*}{$\begin{array}{l}\text { SBT } \\
\text { Category }\end{array}$} & 14 days $(n=204)$ & 30 days $(n=119)$ & 90 days $(n=136)$ \\
\hline & OR $(95 \% \mathrm{Cl})$ & OR $(95 \% \mathrm{Cl})$ & OR $(95 \% \mathrm{Cl})$ \\
\hline Low & 1.0 & 1.0 & 1.0 \\
\hline Medium & $1.0(0.5$ to 2.1$)$ & 1.3 (0.5 to 3.6$)$ & 1.5 (0.5 to 4.2 ) \\
\hline High & 1.7 (0.8 to 3.6$)$ & 1.6 (0.6 to 4.5$)$ & 1.4 (0.4 to 4.3$)$ \\
\hline
\end{tabular}

$B Q=$ Bournemouth Questionnaire; $S B T=$ Start Back Tool.

risk group. This would mean that all groups in this study may have received the treatment reserved in the Hill et al. study (20) exclusively for the high risk group, thus masking any initial prognostic information given by the SBT at baseline. However, any treatment effects must be viewed as strongly speculative due to this study's purely observational design.

It has been suggested that patients presenting to chiropractors are a psychologically healthy subgroup with few individuals having levels of adverse psychological factors sufficient to influence their prognosis [24]. In support of this, a narrative review comparing reports of psychological questionnaires applied to chiropractic populations and non-chiropractic populations where the same test tools have been used [36], concluded that those seeing chiropractors were less likely to have adverse scores across a range of psychological domains that have previously been linked to poor prognosis in other LBP populations. In this study $26.5 \%$ of patients were categorised by the SBT as in the high-risk group. This suggests that either a significant proportion had potentially adverse psychology, or that the SBT is inappropriately categorizing some of those in this population.

The failure of SBT categorisation in this LBP population to identify those less likely to improve may be because, as a self-selecting subgroup, these individual possess features that negate the impact of otherwise adverse psychology. The possible existence of 'protective' factors in some populations is supported by the presence of higher pain related self-efficacy reducing the impact of raised fear avoidance beliefs in patients with chronic LBP [37]. Reports from one chiropractic study that has looked at this found more favorable levels of self-efficacy than other reported populations, and higher levels of self-efficacy were found to relate to a better prognosis $[26,36]$.

Alternatively a reduction in levels of adverse psychological variables may have occurred during care, therefore reducing their effect on treatment response. In support of this, a systematic review of psychological outcomes from studies involving manipulation describes significantly greater reduction in adverse scores on psychological questionnaires in populations having manipulation when compared to groups receiving verbal interventions (advice, education or handout) or other physical treatments (exercise, electrotherapy, sham manipulation or acupuncture) [38]. In addition to this, a small study found that a statistically significant reduction in fear avoidance beliefs and catastrophisation as well as improvement in self-efficacy occurred shortly after an initial visit with a chiropractor. Despite baseline levels of these variables not relating to self-reported outcome at one month, post-visit scores did display a weak but significant relationship to outcomes, with those retaining two or more higher variables post-visit having increased odds of a poor outcome [26].

Caution however, must be used when interpreting these findings. Firstly, this was not a clinical trial investigating treatment effects and as such it is not possible to ascertain any treatment impact on changes in patients' psychology or symptoms. In addition this population of patients had been drawn from a group of six linked practices and as such may not be representative of the wider population seeking privately funded treatment for LBP.

Table 8 Adjusted models for predicting poor outcome (PGIC) at 14, 30 and 90 days follow up

\begin{tabular}{|c|c|c|c|c|}
\hline Follow up point & Variables in the equation & OR $(95 \% \mathrm{Cl})$ & Nagelkerke* & sn/sp \\
\hline \multirow[t]{2}{*}{14 days $(n=235)$} & Pain for $>30$ days in year & $3.2(1.9$ to 5.6$)$ & 0.26 & $90 / 25$ \\
\hline & Pain (for every 1 point increase) & 0.8 (0.8 to 0.9$)$ & & \\
\hline \multirow[t]{4}{*}{30 days $(n=131)$} & Duration & & 0.32 & $92 / 29$ \\
\hline & $<1$ month & 1.0 & & \\
\hline & $1-3$ months & $5.6(1.8$ to 17.0$)$ & & \\
\hline & $>3$ months & $2.4(1.1$ to 5.5$)$ & & \\
\hline
\end{tabular}

90 days $(n=150)$

Duration

$$
\begin{array}{ll}
<1 \text { month } & 1.0 \\
1-3 \text { months } & 9.3(3.0 \text { to } 29.0) \\
>3 \text { months } & 3.0(1.3 \text { to } 7.0)
\end{array}
$$

\footnotetext{
* = variance explained by model; $s n=$ sensitivity; $s p=$ specificity; $P G I C=$ Patient Global Impression of Change.
} 
Additionally, there are some differences in response rates between the SBT categories, particularly at 90 days and the potential remains for selection bias through differential attrition. However, comparisons of baseline demographics (age, pain, duration and BQ score) for each of the three SBT categories demonstrated no statistically significant differences between responders and non responders, as was the number of treatments received at 90 days. Although this cannot rule out the possibility that those responding experienced different outcomes to non responders it provides some support for the contention that responders were more likely to be a representative sample based upon care received and baseline factors.

Lastly, comparisons of the results here with other studies are problematic due to differences in follow up times, outcome instruments and other methodological differences.

\section{Conclusion}

This study has shown that LBP patients seeking treatment for chiropractic and categorised by SBT at baseline show no differential risk of poor outcome between categorisation groups. Although this tool does differentiate LBP patients in terms of baseline pain and baseline total BQ scores, these differences disappeared by 30 days. At the present time, it is unclear whether the SBT is transferable to LBP populations outside of those it was originally developed for.

\section{Competing interests}

The author(s) declare that they have no competing interests.

\section{Authors' contribution}

JF conceived of the study, and was involved with its design, data collection, statistical analysis, interpretation and drafting the manuscript. DN performed the statistical analysis and was involved with its interpretation and in drafting the manuscript. Both authors read and approved the final manuscript.

\section{Author details}

${ }^{1}$ Private practice Back2Health, 2 Charles Street, Petersfield, Hants, UK. ${ }^{2}$ Anglo-European College of Chiropractic, 13-15 Parkwood Rd, Bournemouth, UK.

Received: 21 December 2011 Accepted: 5 June 2012

Published: 12 June 2012

\section{References}

1. Hillman M, Wright A, Rajaratnam G, Tennant A, Chamberlain MA: Prevalence of low back pain in the community: implications for service provision in Bradford, UK. J Epidemiol Community Health 1996, 50(3):347-352.

2. Waxman R, Tennant A, Helliwell P: Community survey of factors associated with consultation for low back pain. BMJ 1998, 317 (7172):1564-15647.

3. Croft PR, Macfarlane GJ, Papageorgiou AC, Thomas E, Silman AJ: Outcome of low back pain in general practice: a prospective study. BMJ 1998, 316 (7141):1356-1359.

4. Dunn K: Classification of low back pain in primary care: using "bothersomeness" to identify the most severe cases. Spine 2005, 30 (16):1887-1892.

5. Hestbaek L, Leboeuf-Yde C, Manniche C: Low back pain: what is the longterm course? A review of studies of general patient populations. Eur Spine J 2003, 12(2):149-165.
6. Henschke N, Maher CG, Refshauge KM, Herbert RD, Cumming RG, Bleasel J, et al: Prognosis in patients with recent onset low back pain in Australian primary care: inception cohort study. BMJ 2008, 337:171.

7. Bouter LM, Pennick V, Bombardier C: Cochrane back review group. Spine 2003, 28(12):1215.

8. vanTulder M, Becker A, Bekkering T, Breen A, Gil del Real M, Hutchinson A et al: European guidelines for the management of acute nonspecific low back pain in primary care. Eur Spine J 2006, 15:169-15. 16.

9. Chou R, Quseem A, Snow V, Casey D, Cross T, Shekelle P, et al: Diagnosis and Treatment of Low Back Pain: A Joint Clinical Practice Guideline from the American College of Physicians and the American Pain Society. Ann Intern Med 2007, 2:147-478. 147.

10. NICE, Savigny P, Watson P, Underwood MR, Guideline Development Group: Early management of persistent non-specific low back pain: summary of NICE guidance. BMJ 2009, 338.

11. Jellema P, Van Der Windt D: Prediction of an unfavourable course of low back pain in general practice: comparison of four instruments. $\mathrm{Br} \mathrm{J}$ General Practice 2007, 57(534):15-22.

12. Melloh M, Elfering A, EgliPresland C, Roeder C, Barz T, RolliSalathh C, et al: Identification of prognostic factors for chronicity in patients with low back pain: a review of screening instruments. International Orthopaedics (SICOT) 2009, 33(2):301-301.

13. Leboeuf-Yde C, Greboeuf-Y A, Borge JA, Lothe J, Magnesen E, Nilsson Nilssoal: The nordic back pain subpopulation program: demographic and clinical predictors for outcome in patients receiving chiropractic treatment for persistent low back pain. JMPT 2004, 27(8):493-502.

14. Newell D, Field J: Who will get better? Predicting clinical outcomes in a chiropractic practice. Clin Chiropr 2008, 13(1):108.

15. Lakke S, Soer R, Takken T, Reneman M: Risk and prognostic factors for nonspecific musculoskeletal pain: A synthesis of evidence from systematic reviews classified into ICF dimensions. Pain 2009, 147(3):153-164.

16. Chou R, Shekelle P: Will this patient develop persistent disabling low back pain? JAMA 2010, 303(13):1295-1302.

17. Ramond A, Bouton C, Richard I, Roquelaure Y, Baufreton C, Legrand E, et al: Psychosocial risk factors for chronic low back pain in primary care--a systematic review. Fam Pract 2011, 28(1):12-21.

18. Thomas E, Silman AJ, Croft PR, Papageorgiou AC, Jayson MIV, Macfarlane GJ: Predicting who develops chronic low back pain in primary care: a prospective study. BMJ 1999, 318:1662-1667.

19. Bolton JE, Hurst $\mathrm{H}$ : Prognostic factors for short-term improvement in acute and persistent musculoskeletal pain consulters in primary care. Chiropr Man Therap 2011, 19:27.

20. Hill JC, Dunn KM, Lewis M, Mullis R, Main CJ, Foster NE, et al: A primary care back pain screening tool: identifying patient subgroups for initial treatment. Arthritis Rheum 2008, 59(5):632-641.

21. Celestin J, Edwards RR, Jamison RN: Pretreatment Psychosocial Variables as Predictors of Outcomes Following Lumbar Surgery and Spinal Cord Stimulation: A Systematic Review and Literature Synthesis. Pain Medicine 2009, 10(4):639-653.

22. Kamper SJ, Maher CG, Hancock MJ, Koes BW, Croft PR, Hay EM: Treatmentbased subgroups of low back pain: a guide to appraisal of research studies and a summary of current evidence. Best Practice \& Research Clinical Rheumatology 2010, 24(2):181-191.

23. Grotle M, Foster NE, Dunn KM, Croft P: Are prognostic indicators for poor outcome different for acute and chronic low back pain consulters in primary care? Pain, 2010, 151(3):790-797.

24. Langworthy J, Breen A: Psychosocial factors and their predictive value in chiropractic patients with low back pain: a prospective inception cohort study. ChiroprOsteopat 2007, 15:5

25. Leboeuf-Yde C, Rosenbaum A, Axen I, Lövgren P, Jørgensen K, Halasz L, et al: The Nordic Subpopulation Research Programme: prediction of treatment outcome in patients with low back pain treated by chiropractors - does the psychological profile matter? ChiroprOsteopat 2009, 17:14.

26. Field J, Newell D, McCarthy PW: Preliminary study into the components of the fear-avoidance model of LBP: change after an initial chiropractic visit and influence on outcome. ChiroprOsteopat 2010, 18:21.

27. Hill JC, Whitehurst DG, Lewis M, Bryan S, Dunn KM, Foster NE, et al: Comparison of stratified primary care management for low back pain with current best practice (STarT Back): a randomised controlled trial. Lancet 2011, 378(9602):1560-1571. 
28. Kongsted A, Johannesen E, Leboeuf-Yde C: Feasibility of the STarT Back Screening Tool in chiropractic clinics: A cross-sectional study of patients with low back pain. Chiropr Man Therap 2011, 19:10

29. Bolton JE, Breen A: The Bournemouth Questionnaire: a short-form comprehensive outcome measure. I. Psychometric properties in back pain patients. JMPT 1999, 22(8):503-510

30. Hill JC, Vohora K, Dunn KM, Main CJ, Hay EM: Comparing the STarT back screening tool's subgroup allocation of individual patients with that of independent clinical experts. The Clinical journal of pain 2010, 26(9):783-787.

31. Newell D, Bolton JE: Responsiveness of the Bournemouth Questionnaire in determining minimal clinically important change in subgroups of low back pain patients. Spine 2010, 35(19):1801-1806.

32. Ostelo RW, Deyo RA, Stratford P, Waddell G, Croft P, Von Korff M, Bouter LM de Vet HC: Interpreting change scores for pain and functional status in low back pain: towards international consensus regarding minimal important change. Spine 2008, 33(1):90-94.

33. Hurst $\mathrm{H}$, Bolton J: Assessing the clinical significance of change scores recorded on subjective outcome measures. I Manipulative PhysiolTher 2004, 27(1):26-35.

34. Extension of Patient choice of Any Qualified Provider in MSK Services for Back and Neck Pain. http://www.supplyzhealth.nhs.uk.

35. Fritz JM, Beneciuk JM, George SZ: Relationship Between Categorization With the STarT Back Screening Tool and Prognosis for People Receiving Physical Therapy for Low Back Pain. Phys Ther 2011, 91(5):722-732.

36. Field J, Newell D, McCarthy P: Are chiropractic patients a psychologically self defining subgroup? Clin Chiropr 2011, 14(2):72-73.

37. Woby SR, Urmston M, Watson PJ: Self-efficacy mediates the relation between pain-related fear and outcome in chronic low back pain patients. Eur J Pain 2007, 11(7):711-718.

38. Williams $\mathrm{N}$, Hendry M, Lewis R, Russell I, Westmoreland A, Wilkinson C: Psychological response in spinal manipulation (PRISM): A systematic review of psychological outcomes in randomised controlled trials. Complementary Therapies in Medicine 2007, 15(4):271-283.

doi:10.1186/2045-709X-20-17

Cite this article as: Field and Newell: Relationship between STarT Back Screening Tool and prognosis for low back pain patients receiving spinal manipulative therapy. Chiropractic \& Manual Therapies 2012 20:17.

\section{Submit your next manuscript to BioMed Central and take full advantage of:}

- Convenient online submission

- Thorough peer review

- No space constraints or color figure charges

- Immediate publication on acceptance

- Inclusion in PubMed, CAS, Scopus and Google Scholar

- Research which is freely available for redistribution 